\title{
Subject Index Vol. 222, 2008
}

\section{Ophthalmologica}

Acute zonal occult outer retinopathy 149

Adalimumab 292

Adult-onset foveomacular vitelliform dystrophy 240

Adverse side effects 292

Age-related macular degeneration 117, $199,321,364$

- - -, animal model 254

- maculopathy 378

Alkaline burn 178

Allergic conjunctivitis, combined therapy 232

Anophthalmic orbit 351

Antibiotic susceptibility 157

Antigen-presenting cells 272, 369

Anti-VEGF 321

Apoptosis 369

Aqueous humor 324

Artisan ${ }^{\circledR} 69$

Autofluorescence 240

Axial length 397

Bacterium 245

Bayliss effect 373

Behcet uveitis 344

Beijing Eye Study 378

Benign ocular adnexal tumour 136

Bimatoprost 88,161

Bleb grading 408

Bone marrow infiltration 422

Buried suture technique 100

Cataract 96

- surgery 130

Chemical mediator release inhibitor 232

Children 329

Chinese patients 149

Choroidal neovascularization 117, 254, 364

Chromium 324

Chromovitrectomy 123

Clinical studies 173

$\mathrm{CO}_{2}$ laser 74

Cocaine use, history 296

Concentration 205

Conjunctiva 136, 338

Conjunctival autograft 105, 265

Cornea 308, 369

Corneal resistance factor 334

- wound healing 380

Costimulatory pathway 272

Curcumin 178

Cysteine 380

Vol. 222, No. 1, 2008 has its own Subject Index

Deep sclerectomy 74, 81

Demarcation line 220

Dengue fever 317

Depression 205

Diabetes 64

- mellitus 168, 225, 284, 324, 373

Diabetic cataract 324

- macular edema 249

- retinopathy 225,284

Diplopia 225

Disruption of outer retina 317

Efficacy 414

Endophthalmitis 157, 357

Erbium:YAG laser 74

Excimer laser 74

- - photoablation 380

Exenteration 338

Eye affection 292

Eyelid crease 100

Fibrin sealant 105

Filtering bleb 408

Flow rates 96

Foveolitis 317

Fungus 245

Glaucoma 161, 400

- surgery 81, 408

Glycosylated haemoglobin 168

Guiding hyaloidal structures 220

Health portal accessibility 187

Heidelberg retina tomograph 168

High myopia 130

Histamine $\mathrm{H}_{1}$ antagonist 232

Holladay II formula 302

Human corneal endothelial cells 272

Humphrey Field Analyser 400

- Matrix Perimeter 400

Hysteresis 334

Immune response 249

Indocyanine green 123

Internal limiting membrane 123

Internet, health information 187

Intraocular lens 397

- - calculation 302

- pressure $81,88,112,414$

Intravascular papillary endothelial hyperplasia 213

Intravitreal injection 364
LASEK, pain 229

Laser in situ keratomileusis 69

- spot 64

Levator function 351

Linoleic acid hydroperoxide 254

Lipofuscin 240

Local anaesthesia, history 296

Lymphangiogenesis, rat, human 308

Macrohematuria 422

Macular burn 117

- hole 123

Maculopathy 317

Magnetic resonance imaging 397

Malignant tumors 338

Masson's tumor 213

Meningitis 357

Metabolic control 373

Mini-flap 265

Minimally classic neovascular membrane 321

Mitomycin C 81, 265

Mortality 378

Multiport illumination system 145

Myogenic response 373

Myxoma 136

Nd:YAG laser 130

Neovascularization 178

Neurogenic blepharoptosis 360

Non-penetrating glaucoma surgery 74

Nuclear factor- $\mathrm{B} 178$

Occult neovascular membrane 321

Ocular aberrations 334

- circulation 88

Ophthalmology, history 296

Ophthalmoplegia 225

Optic capture 302

- disc topography 168

- neuropathy 292

Optical coherence tomography 240

Orbital tumor 213

- wall reconstruction, complication 360

Overgrowing epithelial cells 220

Oxidative stress 194

Paediatric cataract 302

Pars plana vitrectomy 277

Periocular hemorrhage 422

Peripheral blood mononuclear cells 272

Phacoemulsification 96, 112, 277

Phakic intraocular lens 69 
Photorefractive keratectomy 386

Posterior segment 145

Predictability 302

Proliferation of retinal vessels 220

Proliferative vitreoretinopathy 357

Prosthesis 351

Pseudoangiosarcoma 213

Pseudoexfoliation syndrome 112

Psychological distress 199

Pterygium 105, 265, 391

- recurrence 391

Ptosis 360

Pyruvate 194

Quality assessment 187

- evaluation 187

Ranibizumab 321

Reactive oxygen species 194

Refraction 386

Refractive surgery 69, 380

Regression of vasa hyaloidea 220

Reinjection 364

Reproducibility 64

Restasis 391

Retained lens fragments 277

Retinal arterioles 373

- damage 194

- detachment 130

- nerve fibre layer thickness 168

- pigment epithelium 254

- _ - atrophy 117

- vessel analyzer 373
Retinopathy of prematurity 220

Rhabdomyosarcoma 422

Riboflavin pretreatment 369

Serum 324

Silicone oil 245

SITA Fast 329

Stability 386

Staphylococcus 157

Streptococcus pneumoniae 357

Subretinal hemorrhage 199

Sub-Tenon's anesthesia 414

Suture 105

T helper cells 249

Tear film 161

- - function 284

Th1/Th2 balance 249

TNF- $\alpha$ antagonists 292

Topical cyclosporine A 391

Trabeculectomy 81, 408

Transgenic mice 380

Transplantation 308

Transpupillary thermotherapy 117

Travoprost 88, 161

Ultraviolet irradiation 369

Upper eyelid 100

Vacuum 96

Vascular endothelial growth factor 178

- _ _ _ inhibitors 364

Viscoelastic 229
Vision, methodology 173

Vision-related functioning 199

Visthesia 229

Visual acuity 130, 173, 321, 344

- -, computer-based tests 364

- field 64, 400

- $\quad$ - testing strategy 329

- impairment, quality of life 205

- prognosis 344

Visually impaired user 187

Vitrectomy 145, 157

Vitreoretinal surgery 357, 414

Web Accessibility Initiative 187

Well-being 205

Yellow-orange lesion at fovea 317 